\title{
Discrete survival model analysis of a couple's smoking pattern and outcomes of assisted reproduction
}

Jose C. Vanegas', Jorge E. Chavarro 1,2,3, Paige L. Williams 2,4, Jennifer B. Ford ${ }^{6}$, Thomas L. Toth ${ }^{5}$, Russ Hauser $2,5,6$ and Audrey J. Gaskins ${ }^{1,3^{*}}$

\begin{abstract}
Background: Cigarette smoking has been associated with worse infertility treatment outcomes, yet some studies have found null or inconsistent results.

Methods: We followed 225 couples who underwent 354 fresh non-donor assisted reproductive technology (ART) cycles between 2006 and 2014. Smoking history was self-reported at study entry. We evaluated the associations between smoking patterns and ART success using multivariable discrete time Cox proportional hazards models with six time periods: cycle initiation to egg retrieval, retrieval to fertilization, fertilization to embryo transfer (ET), ET to implantation, implantation to clinical pregnancy, and clinical pregnancy to live birth to estimate hazard ratios (HR) and $95 \% \mathrm{Cls}$. Time-dependent interactions between smoking intensity and ART time period were used to identify vulnerable periods.
\end{abstract}

Results: Overall, $26 \%$ of women and 32\% of men reported ever smoking. The HR of failing in the ART cycle without attaining live birth for male and female ever smokers was elevated, but non-significant, compared to never smokers regardless of intensity ( $\mathrm{HR}=1.02$ and 1.30 , respectively). Female ever smokers were more likely to fail prior to oocyte retrieval (HR: 3.37; $95 \% \mathrm{Cl}: 1.00,12.73)$. Every one cigarette/day increase in smoking intensity for females was associated with a HR of 1.02 of failing ART $(95 \% \mathrm{Cl}: 0.97,1.08)$, regardless of duration or current smoking status. Women with higher smoking intensities were most likely to fail a cycle prior to oocyte retrieval (HR: 1.07; 95\% Cl: 1.00, 1.16). Among past smokers, every additional year since a man had quit smoking reduced the risk of failing ART by $4 \%$ (HR: 0.96; 95\% Cl: 0.91, 1.00) particularly between clinical pregnancy and live birth (HR: 0.86; 95\% Cl: 0.76, 0.96).

Conclusions: Female smoking intensity, regardless of current smoking status, is positively associated with the risk of failing ART cycles between initiation and oocyte retrieval. In men who ever smoked, smoking cessation may reduce the probability of failing ART, particularly between clinical pregnancy and live birth.

Trial registration: NCT00011713. Registered: 27 February 2001.

Keywords: Smoking, Fertility, in vitro fertilization, Assisted reproductive technologies, Reproductive health, Survival analysis

\footnotetext{
* Correspondence: audrey.gaskins@mail.harvard.edu

${ }^{1}$ Department of Nutrition, Harvard T.H. Chan School of Public Health, 665 Huntington Ave., 02115 Boston, MA, USA

${ }^{3}$ Channing Division of Network Medicine, Department of Medicine, Brigham and Women's Hospital and Harvard Medical School, 181 Longwood Ave., 02115 Boston, MA, USA

Full list of author information is available at the end of the article
} International License (http://creativecommons.org/licenses/by/4.0/), which permits unrestricted use, distribution, and reproduction in any medium, provided you give appropriate credit to the original author(s) and the source, provide a link to the Creative Commons license, and indicate if changes were made. The Creative Commons Public Domain Dedication waiver (http://creativecommons.org/publicdomain/zero/1.0/) applies to the data made available in this article, unless otherwise stated. 


\section{Background}

Tobacco is among the leading causes of death and disability worldwide [1], with over 6 million people dying of tobacco-related causes per year [2]. It is a common exposure in the US, with smoking reported for approximately $25 \%$ of reproductive-aged women, $12 \%$ of women during pregnancy [3], and $25 \%$ of men aged 25 to 44 years [4]. Cigarette smoke contains about 4000 compounds that have been associated with increased risk of cardiovascular disease, lung disease, cancer $[5,6]$ and adverse reproductive outcomes [7, 8].

Previous studies have shown that a couple's likelihood of success when undergoing assisted reproduction varies depending on their smoking history [9]. Female smoking has been associated with poorer ovarian response markers, lower oocyte counts, and lower rates of implantation [10] and live birth [11, 12] following assisted reproductive technology (ART). Male smoking has also been associated with lower live birth rates [12], independent of female smoking. To date, most studies have been limited in their assessment of smoking history, generally comparing "smokers" to "non-smokers" without making clear distinctions among past and never smokers and rarely taking into account smoking intensity, duration or cessation. Furthermore none of the previous studies have utilized methods which allow distinction of smoking effects by stage of the ART cycle, and thus have not always been able to account for early failures, which may have resulted in contradictory findings [13].

Thus it remains unclear whether characteristics of a couple's smoking history, such as duration and intensity of smoking, the age at smoking initiation, and years since smoking cessation could affect the probability of succeeding at ART. To address this, we evaluated the association of pre-treatment smoking patterns and infertility treatment outcomes in a prospective cohort of couples undergoing ART at the Massachusetts General Hospital (MGH) Fertility Center, using novel statistical methods to allow for a changing effect of smoking on ART failure across time periods within an ART cycle.

\section{Methods}

\section{Study overview}

Participants were couples enrolled in the Environment And Reproductive Health (EARTH) study, an ongoing prospective cohort study started in 2006 aimed at identifying determinants of fertility among couples presenting to the MGH Fertility Center (Boston, MA) [14]. The study was approved by the Institutional Review Boards of MGH and the Harvard T. H. Chan School of Public Health. All participants provided written informed consent.

\section{Study population}

Couples presenting to the MGH Fertility Center were given information about the EARTH Study by the medical team. Study personnel approached couples who met the age requirements (18-46 years for women; 1855 years for men), and who initially planned to use their own gametes for treatment. Approximately $60 \%$ of those contacted participated in the study. Participants were included in the main analysis if they underwent at least one fresh non-donor ART cycle between 2006 and 2014 $(n=392)$. Due to our interest in smoking patterns among couples, we excluded 156 women whose partner had not yet enrolled or elected not to enroll in the EARTH study. Some aspects differed in the excluded couples compared to those included. On average, the female partners from the excluded couples were slightly older (36 vs. 35 years), had higher BMIs (25 vs.24 kg/ $\mathrm{m}^{2}$ ), had more pack-years of smoking (1.4 vs. 0.7 packyears), and were less likely to have completed at least a college education (78\% vs. $86 \%)$. The cycles from the excluded couples were also more likely to fail prior to embryo transfer (15\% vs. $8 \%)$. After exclusions, 236 couples who underwent a total of 354 fresh non-donors ART cycles were included in the analysis.

\section{Smoking assessment}

Participants were asked to report whether they had ever smoked cigarettes (defined as smoking at least 1 cigarette/day for a year or at least 20 packs in their lifetime) in a brief staff-administered questionnaire. Individuals reporting a positive smoking history were asked about their age at initiation, current smoking status, smoke inhalation practices, and the brand of cigarette they used (including size, tobacco type, and filter). Couples reporting current use reported what day they had their last cigarette, how many cigarettes they currently smoke per day, average number of cigarettes smoked per day over their years as a current smoker, and whether they had ever quit for 6 months or more and, if yes, how many years they quit. Former users reported how old they were they quit smoking, if they quit in the last year, the date, quantity of cigarettes smoked per day during the period they smoked, and if before they quit there were periods ( $\geq 6$ months) when they didn't smoke. The total smoking duration was calculated in current users by subtracting the age of smoking initiation from their current age. In former smokers we subtracted the age of smoking initiation from their age on cessation. For both we subtracted their interim cessation periods from their total smoking duration. Pack-years of smoking was calculated as: $\frac{\text { Average number of cigarettes smoked a day }}{20 \text { cigarettes per pack }} \times$ Total smoking duration (years) [15]. Questions on the use of other tobacco products (i.e. cigars, pipes, chewing tobacco and snuff or dip tobacco) 
were included on the questionnaire, but due to their infrequent use in our sample $(<2 \%$ for cigars and $<1 \%$ for the rest), only cigarettes smoking was considered as an exposure in our analyses.

\section{Covariates}

At enrollment, a brief nurse-administered questionnaire was used to collect data on demographic characteristics, medical history, and lifestyle factors. A trained research study staff member measured each participant's height and weight.

\section{ART Outcomes}

Women underwent a pretreatment cycle of oral contraceptives for 2-5 weeks to suppress ovulation before their ART cycles, unless contraindicated. On day 3 of induced menses, patients began controlled ovarian stimulation. Women underwent one of three stimulation protocols as clinically indicated: 1) luteal-phase $\mathrm{GnRH}$ agonist protocol; 2) follicular-phase GnRH-agonist/flare protocol; or 3) GnRH-antagonist protocol. Patients were monitored during gonadotropin stimulation for serum estradiol and endometrial thickness through 2 days before egg retrieval. Human chorionic gonadotrophin was administered $36 \mathrm{~h}$ before the scheduled egg retrieval procedure to induce ovulation. Details of oocyte retrieval have been previously described [16]. Embryologists classified oocytes as germinal vesicle, metaphase I, metaphase II (MII), or degenerated. Following retrieval, couples underwent conventional insemination or intracytoplasmic sperm injection (ICSI) for fertilization. We defined successful implantation as a serum $\beta$-hCG level $>6 \mathrm{mIU} / \mathrm{mL} 15-20$ days after egg retrieval, clinical pregnancy as the presence of an intrauterine pregnancy in ultrasound, and live birth as the birth of a neonate at or after 24 weeks of gestation. All clinical information was abstracted from electronic medical records.

\section{Statistical analysis}

Both male and female participants were classified into three categories: never, former, and current smokers according to self-reported smoking status. We then cross-classified couples into four categories according to their ever smoking status. We evaluated the effect of smoking intensity using the variables average cigarettes/ day and total years of smoking as continuous exposures. Descriptive statistics were calculated for demographic and reproductive characteristics according to current smoking status prior to ART treatment. A Chi-square test or Fisher's exact test was used to test for differences across categories for discrete variables and the KruskalWallis test for differences across categories for continuous variables.
Multivariable Cox proportional hazards models for discrete survival time were used to estimate the hazard ratios (HRs) and 95\% confidence intervals (CIs) for the associations between smoking patterns and risk of failing ART prior to live birth, as proposed by Maity [17] and Missmer [18]. In all of these models, a robust sandwich covariance estimate was used to account for the multiple cycles per woman. HRs estimate the odds of failing an ART cycle at any point, conditional on not failing at an earlier moment during the same cycle. Women were considered at risk of failing ART for the duration of their initiated cycle until their specific point of failure. There were six periods during which women could fail a cycle: between (1) cycle initiation and oocyte retrieval, (2) retrieval and fertilization, (3) fertilization and embryo transfer (ET), (4) ET and implantation, (5) implantation and clinical pregnancy, and (6) between clinical pregnancy and live birth. Estimated probabilities of survival for the average women in our cohort (according to mean or reference levels for covariates other than smoking) were used to construct survival curves across these time points.

Confounding was evaluated through directed acyclic graphs based on prior knowledge and descriptive statistics in the study population. The multivariable models were adjusted for age, BMI, protocol, educational level and partner's smoking history (never or ever smoker). We centered smoking intensity, years of smoking duration and pack-years by the whole sample's mean for continuous analyses. For analysis of smoking history (ever vs. never smoker), smoking status (never, former and current smoker) and couple's cross-classifications, we further adjusted by centered pack-years. We analyzed as continuous variables smoking intensity and duration in the entire population years since smoking cessation and age at smoking initiation among ever smokers (58 women and $71 \mathrm{men})$. Never smokers were assigned a value of 0 average cigarettes/day and 0 years of total smoking for the continuous analyses. Since in Massachusetts insurance companies require current smokers to have cotinine levels below the non-smoker reference value for 2 months to provide authorization for IVF coverage, we assigned a cessation time of 2 months for current smokers. All results are presented as HRs for a 1 unit increase in the continuous exposure. We further adjusted the models for smoking intensity (cigarettes/day) and smoking duration (years) using a binary variable for self-reported current smoking status. Models for smoking cessation and age at initiation were further adjusted for pack-years. The model of age at smoking initiation was also further adjusted for a binary variable for selfreported current smoking-status. To evaluate whether there were specific stages of the cycle which were more vulnerable to the effects of smoking, we fit multivariate 
models with time-dependent interaction between the discrete time periods and smoking exposures.

We also ran a variety of sensitivity analysis to address potential issues related to misclassification of the exposure, selection bias into the study, and model fitting. In accordance with a prior methodological paper on smoking and lung cancer [19], we conducted a sensitivity analysis re-classifying the participant's self-reported smoking status according to their time of cessation in three discrete categories: never smokers (self-reported never smokers), former smokers (self-reported former smokers with $>2$ years of smoking cessation) and "clinically active smokers" (selfreported current smokers and self-reported former smokers with $\leq 2$ years of smoking cessation). Also, in order to assess the possibility of selection bias in our sample (due to the exclusion of women who did not have a partner enrolled), we conducted a sensitivity analysis including all woman regardless of whether their partner was enrolled and analyzed the association between smoking history and probability of failing ART without adjusting for the male partner's smoking history. Finally, we performed an analysis limited to the couple's first cycle to address the potential limitation of this model to account for the imbalanced number of cycles per couple.

To illustrate the possible effect of survival bias, we used generalized linear mixed models with random intercepts to analyze the associations between smoking patterns and oocyte yield (Poisson distribution and log link); endometrial wall thickness and peak serum estradiol at trigger day (binomial distribution and log link) among women who underwent oocyte retrieval, and probability of live birth per initiated cycle and per embryo transfer (binary distribution and logit link). These results are presented as population marginal means adjusted for covariates. All analyses were conducted using the Statistical Analysis System Software package SAS 9.4 (SAS Institute Inc., Cary, NC) and considered two-sided significance levels $<0.05$ as statistically significant.

\section{Results}

Our sample consisted mostly of non-Hispanic Caucasians ( $86 \%$ of women; $88 \%$ of men) with a mean age of 34.8 and 36.6 years for female and male participants, respectively. About $26 \%$ of women were self-reported ever smokers (23\% former and 3\% current smokers); $32 \%$ of men were ever smokers (24\% former and $7 \%$ current smokers). On average, women who ever smoked started smoking when they were 17 years, smoked 6 cigarettes/day for 9 years, and if they had quit, they did so 7.5 years before cycle initiation. Similarly, men who ever smoked started smoking when they were an average of 18 years old, and on average smoked 9 cigarettes per day for 12 years, and if they had quit, they did so 6 years before cycle initiation. Men and women who were current smokers were more likely to have a partner who currently smoked and were less likely to have a college education compared to never smokers (Tables 1 and 2). Current smokers also reported smoking for longer durations compared to former smokers. Current male smoker were more likely to have undergone reproductive tract surgery prior cycle initiation. Current female smokers were more likely to have their first cycle cancelled prior to embryo transfer and had higher baseline FSH levels.

Smoking status of the female partner, the male partner, and the cross-classified couple smoking status were unrelated to the risk of cycle failure (Table 3). The HR of failing ART for female ever smokers compared to never smokers was 1.30 (95\% CI: 0.84, 2.02; P-Value $=0.24$ ) and estimated HRs remained similar even after accounting for smoking intensity and duration. When evaluating the effects of smoking during specific periods of potential ART failure, ever female smokers were marginally more likely to fail prior to oocyte retrieval (HR: 3.57; 95\% CI: 1.00, 12.73; P-Value $=0.05$ ) but not during other periods of the ART cycle. In the self-reported current smoking status analysis, the HR of failing ART for former and current female smokers was 1.23 (95\% CI: 0.79, 1.91; P-Value $=0.35)$ and 2.41 (95\% CI: 0.62, 9.39; $P$-Value $=0.20)$ when compared to never smokers. Similarly, current smokers (HR: 6.0; 95\% CI: 0.96, 37.07; PValue $=0.05)$ and former smokers (HR: $2.05 ; 95 \% \mathrm{CI}$ : $0.50,8.34 ; P$-Value $=0.32$ ) were more likely to fail prior to oocyte retrieval compared to never smokers but this association was not significant for the other ART time points and only borderline significant for current smokers. Women whose male partners were former and current smokers had no increased risk of failing ART when compared to never smokers. In addition, couple classifications in which either or both of the partners ever smoked, as compared to couples in which neither partner ever smoked, did not have an increase in risk of cycle failure.

For the intensity and duration analyses (Table 4), a 1 cigarette/day increase in female smoking intensity was associated with a $7 \%$ increased risk of failing at ART $(\mathrm{HR}=1.07,95 \% \mathrm{CI}: 0.97,1.08$; $P$-Value $=0.45)$ after further adjustment for smoking duration and current smoking status; while a 1 year increase in smoking duration yielded a HR of 1.01 (95\% CI: 0.97, 1.05 P-Value $=0.61$ ) after further adjustment for smoking intensity and current smoking status. Greater intensity of female smoking was associated with a borderline higher risk of failing a cycle prior to oocyte retrieval (HR: 1.07; 95\% CI: $1.00,1.16$; $P$-Value $=0.07$ ); In contrast smoking duration was not significantly related to the probability of failing ART during any period of the ART cycle. Neither time of cessation nor age of smoking initiation was significantly associated with the risk of failing ART (Table 4). 
Table 1 Baseline characteristics of 225 couples from the EARTH study in relation to female smoking status

\begin{tabular}{|c|c|c|c|c|}
\hline & \multicolumn{3}{|c|}{ Female Smoking Status } & \multirow[t]{2}{*}{$P$-value ${ }^{a}$} \\
\hline & Never smoker & Former smoker & Current smoker & \\
\hline Number of couples, n (\%) & $167(74.2)$ & $51(22.7)$ & $7(3.1)$ & $<0.001$ \\
\hline Total ART cycles, n (\%) & $249(70.3)$ & $93(26.3)$ & $12(3.4)$ & $<0.001$ \\
\hline \multicolumn{5}{|l|}{ Female Characteristics ${ }^{b}$} \\
\hline Age, years & $35(32,38)$ & $34(33,37)$ & $34(29,37)$ & 0.89 \\
\hline Body Mass Index, $\mathrm{kg} / \mathrm{m}^{2}$ & $22.6(20.4,26.3)$ & $22.7(21.5,24.7)$ & $25.7(23.8,28.2)$ & 0.11 \\
\hline At least college education, n (\%) & $148(88.6)$ & $39(76.5)$ & $5(71.4)$ & 0.04 \\
\hline Non-Hispanic Caucasian, n (\%) & $143(85.6)$ & $43(84.3)$ & $7(100)$ & 0.74 \\
\hline Pack-years & NA & $1.6(0.5,4.6)$ & $2.4(1.0,4.2)$ & 0.65 \\
\hline Total smoking duration, years & NA & $9(5,13)$ & $14(8,16)$ & 0.08 \\
\hline Average cigarettes smoked & NA & $4.0(1.5,10.0)$ & $5.0(1.5,7.0)$ & 0.89 \\
\hline Age of smoking initiation, years & NA & $17(15,18)$ & $18(18,20)$ & 0.04 \\
\hline Total Smoking cessation, years & NA & $8.0(5.0,12.0)$ & $0.2(0.2,0.2)$ & $<0.001$ \\
\hline Exposed to passive smoking, $\mathrm{n}(\%)$ & $30(18.0)$ & $14(27.5)$ & $2(28.6)$ & 0.23 \\
\hline \multicolumn{5}{|l|}{ Male Partner Characteristics } \\
\hline Partner age, years & $36(33,40)$ & $35(33,40)$ & $39(35,41)$ & 0.40 \\
\hline Partner body mass index, $\mathrm{kg} / \mathrm{m}^{2}$ & $26.9(24.2,29.3)$ & $26.9(24.5,29.3)$ & $27.9(25.1,28.3)$ & 0.97 \\
\hline Partner former smoker, n (\%) & $38(22.8)$ & $16(31.4)$ & $1(14.3)$ & 0.44 \\
\hline Partner current smoker, n (\%) & $8(4.8)$ & $4(7.8)$ & $4(57.1)$ & $<0.001$ \\
\hline \multicolumn{5}{|l|}{ Baseline Reproductive Characteristics } \\
\hline Previous IUI, n (\%) & $71(42.5)$ & 19 (37.3) & $2(28.6)$ & 0.70 \\
\hline Previous IVF, n (\%) & $40(24.0)$ & $8(15.7)$ & $1(14.3)$ & 0.51 \\
\hline ICSI, n (\%) & $83(49.7)$ & $31(60.8)$ & $4(57.1)$ & 0.37 \\
\hline Infertility diagnosis, n (\%) & & & & 0.47 \\
\hline Male factor & $55(32.9)$ & $20(39.2)$ & $4(57.1)$ & \\
\hline Diminished ovarian reserve & $9(5.9)$ & $3(5.9)$ & $1(14.3)$ & \\
\hline Endometriosis & $10(6.0)$ & $3(5.9)$ & $0(0.0)$ & \\
\hline Ovulatory dysfunction & $15(9.0)$ & $5(9.8)$ & $0(0.0)$ & \\
\hline Tubal causes & $11(6.6)$ & $8(15.7)$ & $1(14.3)$ & \\
\hline Uterine causes & $3(1.8)$ & $0(0.0)$ & $0(0.0)$ & \\
\hline Unexplained & $64(38.3)$ & $12(23.5)$ & $1(14.3)$ & \\
\hline Initial treatment protocol, n (\%) & & & & 0.69 \\
\hline Antagonist & $21(12.6)$ & $5(9.8)$ & $1(14.3)$ & \\
\hline Flare & $17(10.2)$ & $8(15.7)$ & $0(0.0)$ & \\
\hline Luteal phase agonist & $129(77.3)$ & $38(75.5)$ & $6(85.7)$ & \\
\hline Day 3 FSH levels, IU/L & $6.6(5.8,8.0)$ & $7.3(6.0,8.8)$ & $8.1(7.0,8.3)$ & 0.16 \\
\hline E2 Trigger levels, pmol/L & $2126(1632,2639)$ & $1828(1459,2532)$ & $1798(1540,1953)$ & 0.17 \\
\hline Day of embryo transfer, n (\%) & & & & 0.01 \\
\hline No transfer & $11(6.6)$ & $4(7.8)$ & $2(28.6)$ & \\
\hline Day 2 & $4(2.4)$ & $5(2.2)$ & $0(0.0)$ & \\
\hline Day 3 & $79(47.6)$ & $29(56.9)$ & $1(14.3)$ & \\
\hline Day 5 & $72(43.4)$ & $13(25.5)$ & $4(57.1)$ & \\
\hline
\end{tabular}


Table 1 Baseline characteristics of 225 couples from the EARTH study in relation to female smoking status (Continued)

$\begin{array}{llcl}\text { Number of embryos transferred, } n(\%) & & <(28.6) \\ \text { No transfers } & 11(6.6) & 4(7.8) & 2(28.6) \\ 1 \text { embryo } & 42(25.3) & 2(3.9) & 3(42.7) \\ 2 \text { embryos } & 81(48.8) & 41(80.4) & 0.001 \\ 3 \text { or more embryos } & 32(19.3) & 4(7.8) & 0.0)\end{array}$

${ }^{\mathrm{a}}$ From Chi-square or Fisher test for discrete variables and Kruskal-Wallis for continuous variables

${ }^{\mathrm{b}}$ Continuous variables are presented as median ( $25^{\text {th }}$ percentile, $75^{\text {th }}$ percentile) while categorical variables are presented as number of women (percent) Abbreviations: ART Assisted reproductive technology, BMI Body mass index, E2 estradiol, FSH follicle stimulating hormone, IVF In-vitro fertilization, ICSI Intracytoplasmic sperm injection, IUI intrauterine sperm insemination

We found a borderline significant protective effect of smoking cessation in ever-smoker males; for every additional year since a male smoker quit, the risk of failing during an ART cycle decreased by $4 \%$ (HR: 0.96; 95\% CI: 0.91, 1.00; P-Value $<0.05)$ regardless of smoking duration and intensity. This association was particularly pronounced for the time period between clinical pregnancy and live birth (HR: 0.86; 95\% CI: 0.76, 0.96; P-Value $=0.01$ ).

Reclassification of smoking status by taking into account time since smoking cessation did not significantly alter our results. For instance, compared to never smokers, former smokers had a $23 \%$ increase in risk $(\mathrm{HR}=1.23,95 \%$ CI: $0.78,1.95 ; \quad P$-Value $=0.37)$ while "clinically active" smokers had a HR of 1.62 (95\% CI: 0.70, 3.78; P-Value $=0.37$ ) of failing at ART; similar to the main analysis, the increased risk of failing at ART was predominantly prior to oocyte retrieval (HR: 2.47; 95\% CI: 1.27, 4.78; P-Value $=0.01$ ) for "clinically active" smokers. The sensitivity analysis including the women without a male partner enrolled in the study provided consistent results to those seem in the main sample. Specifically, female ever smokers had a borderline significant HR of 1.38 (95\% CI: 0.99, 1.92; P-Value $=0.06$ ) compared to never smokers; also, this increased risk was significantly higher for failing prior to oocyte retrieval (HR: 2.45; 95\% CI: 1.12, 5.36; P-Value $=0.02$ ) and it was also significant between embryo transfer and implantation (HR: 1.63; 95\% CI: 1.03, 2.58; P-Value $=0.04$ ). An increase of 1 cigarette/day produced an overall HR of 1.01 (95\% CI: 0.97, 1.06; P-Value $=0.65)$ for failing at ART and the HR was not significantly different according to the particular moment of the cycle. Finally, sensitivity analyses limited to the couple's first cycle produced results that were consistent with the main analysis, although with loss of significance given the reduction in power: female ever smokers had an HR of 1.34 (95\% CI: 0.76, 2.34; P-Value $=0.31$ ) of failing ART compared to never smokers and this increased risk was more pronounced prior to oocyte retrieval (HR: 3.00; 95\% CI: 0.56, 16.08; P-Value $=0.20$ ) although these increases were not statistically significant.

Given the observed relation between female smoking and early ART failures, we analyzed the associations between smoking patterns and oocytes counts among women with successful egg retrieval using conventional methods to illustrate the possible effect of survival bias. In these models, self-reported current smokers appeared to have a higher number of total and MII phased oocytes retrieved than former and never smokers even after multivariable adjustment, although these differences did not attain statistical significance (Additional file 1: Table S1); however, $16.7 \%$ of cycles from self-reported current smokers could not be included in this analysis because they failed prior to retrieval compared to only $2.0 \%$ of cycles from never smokers and 6.5\% from former smokers (Additional file 1: Table S1). We did not observe associations with endometrial thickness or peak estradiol levels. When we analyzed the associations between smoking patterns and live birth following ART among the cycles of women undergoing an embryo transfer and all initiated cycles, the predicted probability of live birth was the lowest for current smokers in both cases. However, the differences by smoking status for women undergoing an embryo transfer were attenuated compared to those observed taking into consideration all initiated cycles (Additional file 2: Table S2). This was to be expected as $25 \%$ of cycles from self-reported current smokers failed prior to ET compared to only $6.8 \%$ of cycles from never smokers.

\section{Discussion}

In a prospective cohort of couples undergoing infertility treatment with ART, we found that the female smoking intensity was related to a higher risk of cycle failure prior to oocyte retrieval, even after accounting for duration of smoking and current smoking status. Our results suggest that the effects of smoking on ovarian response to controlled ovarian stimulation may persist after smoking cessation. We also found a borderline protective effect for male smoking cessation and cycle failure between clinical pregnancy and live birth in former smokers. Our conventional analysis highlighted the possibility that studies ignoring failures prior to oocyte retrieval for intermediate outcomes and failures prior to embryo transfer for clinical outcomes may be susceptible to survival bias, possibly explaining some inconsistencies in the literature of the 
Table 2 Baseline characteristics of 225 couples from the EARTH study in relation to male smoking status

\begin{tabular}{|c|c|c|c|c|}
\hline & \multicolumn{3}{|c|}{ Male Smoking Status } & \multirow[t]{2}{*}{$P$-value } \\
\hline & Never smoker & Former smoker & Current smoker & \\
\hline Number of couples, n (\%) & $154(68.4)$ & $55(24.4)$ & $16(7.1)$ & $<0.001$ \\
\hline Total ART cycles, n (\%) & $235(66.4)$ & $89(25.1)$ & $30(8.5)$ & $<0.001$ \\
\hline \multicolumn{5}{|l|}{ Male Characteristics ${ }^{\mathrm{b}}$} \\
\hline Age, years & $35(32,40)$ & $37(34,40)$ & $37(33,39)$ & 0.20 \\
\hline $\mathrm{BMI}, \mathrm{kg} / \mathrm{m}^{2}$ & $26.8(24.3,29.1)$ & $27.4(24.3,30.0)$ & $25.6(23.4,28.3)$ & 0.33 \\
\hline At least college education, n (\%) & $111(72.1)$ & $33(60.0)$ & $7(56.3)$ & 0.15 \\
\hline Non-Hispanic Caucasian, n (\%) & $137(89.0)$ & $48(87.3)$ & $13(81.3)$ & 0.58 \\
\hline Pack-years & NA & $3.3(1.1,7.3)$ & $7.0(3.3,8.7)$ & 0.07 \\
\hline Total smoking duration, years & NA & $10(5,14)$ & $17(14,19)$ & 0.001 \\
\hline Average cigarettes smoked & NA & $6.0(3.0,12.0)$ & $9.3(4.8,11.3)$ & 0.68 \\
\hline Age of smoking initiation, years & NA & $18(16,20)$ & $16(15,20)$ & 0.22 \\
\hline Total Smoking cessation, years & NA & $4.0(1.5,7.8)$ & $0.2(0.2,0.2)$ & $<0.001$ \\
\hline Exposed to passive smoking, $\mathrm{n}(\%)$ & $24(15.6)$ & $14(25.5)$ & $7(43.8)$ & 0.01 \\
\hline \multicolumn{5}{|l|}{ Male Reproductive History } \\
\hline History of cryptorchidism, n (\%) & $10(6.5)$ & $2(3.7)$ & $2(12.5)$ & 0.42 \\
\hline History of varicocele, n (\%) & $16(10.4)$ & $4(7.3)$ & $2(12.5)$ & 0.90 \\
\hline History of reproductive surgery, n (\%) & $31(20.1)$ & $8(14.6)$ & $7(43.8)$ & 0.04 \\
\hline \multicolumn{5}{|l|}{ Female Partner Characteristics } \\
\hline Partner age, years & $34(32,38)$ & $35(33,38)$ & $36(33,39)$ & 0.47 \\
\hline Partner BMI, kg/m² & $22.2(20.7,25.4)$ & $23.8(21.3,27.7)$ & $24.8(20.5,29.2)$ & 0.11 \\
\hline Partner former smoker, n (\%) & $31(20.1)$ & $16(29.1)$ & $4(25.0)$ & 0.38 \\
\hline Partner current smoker, n (\%) & $2(1.3)$ & $1(1.8)$ & $4(25.0)$ & $<0.001$ \\
\hline \multicolumn{5}{|l|}{ Baseline Reproductive Characteristics } \\
\hline Previous IUI, n (\%) & $67(43.5)$ & $18(32.7)$ & $7(43.8)$ & 0.37 \\
\hline Previous IVF, n (\%) & $34(77.9)$ & $11(20.0)$ & $4(25.0)$ & 0.90 \\
\hline ICSI, n (\%) & $82(53.3)$ & $27(49.1)$ & $9(56.3)$ & 0.83 \\
\hline Infertility diagnosis, n (\%) & & & & 0.53 \\
\hline Male factor & $55(35.7)$ & $16(29.1)$ & $8(50.0)$ & \\
\hline Diminished ovarian reserve & $8(5.2)$ & $3(5.5)$ & $2(12.5)$ & \\
\hline Endometriosis & $6(3.9)$ & $6(10.9)$ & $1(6.3)$ & \\
\hline Ovulatory dysfunction & $13(8.4)$ & $7(12.7)$ & $0(0.0)$ & \\
\hline Tubal Causes & $13(8.4)$ & $5(9.1)$ & $2(12.5)$ & \\
\hline Uterine Causes & $2(1.3)$ & $1(1.8)$ & $0(0.0)$ & \\
\hline Unexplained & $57(37.0)$ & $17(30.9)$ & $3(18.8)$ & \\
\hline Initial treatment protocol, n (\%) & & & & 0.05 \\
\hline Antagonist & $16(10.4)$ & $5(9.1)$ & $6(37.5)$ & \\
\hline Flare & $18(11.7)$ & $5(9.1)$ & $2(12.5)$ & \\
\hline Luteal phase agonist & $120(77.9)$ & $45(82.8)$ & $8(50.0)$ & \\
\hline Day 3 FSH levels, IU/L & $6.7(5.8,8.0)$ & $6.6(5.8,8.2)$ & $8.2(7.3,9.5)$ & 0.004 \\
\hline E2 Trigger levels, pmol/L & $2076(1560,2678)$ & $2145(1632,2570)$ & $1761(1225,2311)$ & 0.27 \\
\hline
\end{tabular}


Table 2 Baseline characteristics of 225 couples from the EARTH study in relation to male smoking status (Continued)

$\begin{array}{lccc}\text { Day of embryo transfer, } \mathrm{n}(\%) & & 0.90 \\ \text { No transfer } & 12(7.8) & 2(7.3) & 1(6.3) \\ \text { Day } 2 & 7(4.6) & 1(1.8) & 9(6.3) \\ \text { Day } 3 & 71(46.4) & 29(52.7) & 5(36.3) \\ \text { Day } 5 & 63(41.2) & 21(38.2) & 1(6.3) \\ \text { Number of embryos transferred, } \mathrm{n}(\%) & & 2(7.3) & 4(25.0) \\ \text { No transfer } & 12(7.8) & 8(14.6) & 8(50.0) \\ 1 \text { embryo } & 34(22.2) & 34(61.8) & 3(18.8) \\ 2 \text { embryos } & 83(54.3) & 9(16.4) & 0.90 \\ 3 \text { or more embryos } & 24(15.7) & & 3\end{array}$

${ }^{\mathrm{a}}$ From Chi square or Fisher test for discrete variables and Kruskal-Wallis for continuous variables

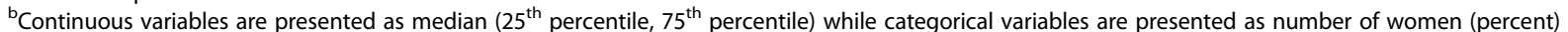
Abbreviations: ART Assisted reproductive technology, BMI Body mass index, E2 estradiol, FSH Follicle stimulating hormone, IVF In-vitro fertilization, ICSI Intracytoplasmic sperm injection, IUI Intrauterine sperm insemination

effects of smoking on human fertility, such as a higher number of oocytes or no differences in clinical outcomes comparing current to never smokers [13].

Despite the low prevalence of current smoking in our cohort, our results do suggest that female smoking is detrimental to overall ART success. These results are in accordance with previous cohort studies and metaanalyses which reported lower probabilities of implantation, clinical pregnancy and live birth following ART in smokers compared to never smokers $[9,12,20,21]$.

Table 3 Estimated cumulative probabilities of live birth and corresponding hazard ratios of failing at ART by discrete smoking patterns (EARTH study, $N=225$ couples, 354 ART cycles)

\begin{tabular}{|c|c|c|c|c|}
\hline & Number of Couples/Cycles & $\begin{array}{l}\text { Estimated cumulative probability } \\
\text { of live birth, per } 100 \text { cycles }^{\mathrm{a}}\end{array}$ & $\begin{array}{l}\text { Adjusted Hazard } \\
\text { Ratio }(95 \% \mathrm{Cl})^{\mathrm{b}}\end{array}$ & $P$-Value \\
\hline Female Smoking History & & & & 0.24 \\
\hline Never Smoker & $167 / 249$ & $54.2(45.8,64.0)$ & 1.00 (REF) & \\
\hline Ever Smoker & $58 / 105$ & $45.0(32.8,61.8)$ & $1.30(0.84,2.02)$ & \\
\hline Female Smoking Status & & & & 0.35 \\
\hline Never Smoker & $167 / 249$ & $54.1(45.7,63.9)$ & 1.00 (REF) & \\
\hline Former Smoker & $51 / 93$ & $46.9(34.5,63.9)$ & $1.23(0.79,1.91)$ & \\
\hline Current Smoker & $7 / 12$ & $22.7(5.6,92.0)$ & $2.43(0.64,9.39)$ & \\
\hline Male Smoking History & & & & 0.93 \\
\hline Never Smoker & $154 / 235$ & $54.0(46.6,68.4)$ & 1.00 (REF) & \\
\hline Ever Smoker & $71 / 119$ & $53.2(43.1,65.7)$ & $1.02(0.64,1.63)$ & \\
\hline Male Smoking Status & & & & 0.75 \\
\hline Never Smoker & $154 / 235$ & $54.3(45.7,64.6)$ & 1.00 (REF) & \\
\hline Former Smoker & $55 / 89$ & $56.4(44.6,71.5)$ & $0.94(0.55,1.61)$ & \\
\hline Current Smoker & $16 / 30$ & $46.7(30.6,72.7)$ & $1.25(0.69,2.25)$ & \\
\hline Couples Ever Smoking Status & & & & 0.59 \\
\hline Female and Male Never Smokers & $121 / 172$ & $54.7(45.7,65.4)$ & 1.00 (REF) & \\
\hline Female Never Smoker, Male Ever Smoker & $46 / 77$ & $50.7(38.4,67.0)$ & $1.12(0.69,1.84)$ & \\
\hline Female Ever Smoker, Male Never Smoker & $33 / 63$ & $42.2(28.7,62.0)$ & $1.43(0.85,2.41)$ & \\
\hline Female and Male Ever Smokers & $25 / 42$ & $47.5(31.8,70.8)$ & $1.24(0.62,2.46)$ & \\
\hline
\end{tabular}

All estimates were calculated from a discrete time Cox proportional Hazard models adjusting for female age (centered continuous) and BMI (continuous); induction protocol (categorical); female educational status (categorical); centered pack-years history (centered continuous) and partner's smoking status (categorical)

${ }^{a}$ Estimates of cumulative probability of not failing ART cycle are given for the average couple in our cohort (e.g. female age (34.8 years) and BMI (23.8 kg/m2); induction protocol (luteal agonist); female educational status (more than college); centered pack-years history ( 0.93 packs-years for women; 2.47 packs-years for men) and partner's smoking status (never smoker)

${ }^{\mathrm{b}} P$-Value is testing for any difference across categories of exposure 
Table 4 Hazard ratios for failing ART by continuous smoking patterns (EARTH study, N=225 couples, 354 ART cycles)

\begin{tabular}{|c|c|c|}
\hline Smoking Pattern & Adjusted Hazard Ratio (95\% Cl) & $P$-Value \\
\hline \multicolumn{3}{|l|}{ Female Smoking Intensity and Duration ${ }^{a}$} \\
\hline Per 1 additional cigarette per day & $1.02(0.97,1.08)$ & 0.45 \\
\hline Per 1 additional year of smoking & $1.01(0.97,1.05)$ & 0.68 \\
\hline \multicolumn{3}{|l|}{ Female Smoking Cessation ${ }^{b}$} \\
\hline Per 1 additional year of smoking cessation & $1.01(0.95,1.08)$ & 0.69 \\
\hline \multicolumn{3}{|l|}{ Female Age of Smoking Initiation ${ }^{c}$} \\
\hline Per 1 additional year later smoking initiation & $0.90(0.79,1.02)$ & 0.11 \\
\hline \multicolumn{3}{|l|}{ Male Smoking Intensity and Duration ${ }^{a}$} \\
\hline Per 1 additional cigarette per day & $0.99(0.97,1.01)$ & 0.36 \\
\hline Per 1 additional year of smoking & $1.01(0.99,1.04)$ & 0.25 \\
\hline \multicolumn{3}{|l|}{ Male Smoking Cessation ${ }^{b}$} \\
\hline Per 1 additional year of smoking cessation & $0.96(0.91,1.00)$ & 0.05 \\
\hline \multicolumn{3}{|l|}{ Male Age of Smoking Initiation ${ }^{c}$} \\
\hline Per 1 additional year later smoking initiation & $1.04(0.91,1.19)$ & 0.58 \\
\hline \multicolumn{3}{|c|}{$\begin{array}{l}\text { Included all couples and cycles ( } N=354 \text { cycles). Data is presented as the cumulative hazard ratio for failing ART at any point per } 1 \text { unit increase of smoking } \\
\text { intensity (continuous, centered average cigarettes/day) and total years of smoking (continuous, centered years). The model was adjusted for female age and BMI, } \\
\text { protocol, educational status, current smoking status and partner's smoking status } \\
\text { bimited to ever smokers only ( } N=105 \text { cycles for women; } N=119 \text { cycles for men). Data is presented as the cumulative hazard ratio for failing ART at any point } \\
\text { per } 1 \text { unit increase of smoking cessation (centered, continuous years). The model was adjusted for female age and BMI, protocol, educational status, pack-years } \\
\text { history and partner's smoking status } \\
\text { cLimited to ever smokers only ( } N=105 \text { cycles for women; } N=119 \text { cycles for men). Data is presented as the cumulative hazard ratio for failing ART at any point per } \\
1 \text { unit increase of smoking age at initiation (centered, continuous years). The model was adjusted for female age and BMI, protocol, educational status, pack-years } \\
\text { history, current smoking status and partner's smoking status }\end{array}$} \\
\hline
\end{tabular}

Similar to our findings, many of these previous studies also found that female smokers were more likely to fail before an embryo transfer [12, 21, 22]. The higher odds of failing between cycle initiation and oocyte retrieval could imply that cigarette smoke disrupts the response to controlled ovarian hyper stimulation protocols or disrupts the ovary itself. This mechanism could be related to findings in animal models, in which cigarette smoke has been found to alter the serum levels of sex hormones and gametogenesis [23, 24]. Although this mechanism accounts only for contemporaneous exposures there seems to be a relation between lower fecundity and cumulative smoking exposure in epidemiological studies [25]. Other epidemiological studies have shown that current smokers were more likely to have worse ovarian stimulation outcomes compared to never smokers [26, 27]. Our findings also suggest an increased risk of failure at implantation that may have been underpowered in our study, as further evidenced by the results of the second sensitivity analysis which included a larger sample of women.

Not all epidemiological studies have found negative associations between smoking and ART success $[13,28]$. Although differences in study design and power may have influenced these results, these studies also tended to analyze clinical outcomes only among cycles reaching the point of embryo transfer. Due to the effects of smoking on early ART outcomes (e.g. response to controlled ovarian stimulation), restricting analyses to only cycles with embryo transfer creates a selection bias in which only the women who survived until then are included, biasing the observed burden of smoking downwards [29] even despite the well-recognized associations between smoking and miscarriage, stillbirth and neonatal death [30]. When we analyzed the association between smoking patterns and live birth per embryo transfer (as opposed to per initiated cycle; Additional file 2: Table S2), we illustrated how this issue may be at play. We also found paradoxical results when we evaluated the association between smoking patterns and oocyte counts among women with successful egg retrieval, as current smokers appeared to yield as many oocytes compared to former and never smokers. However, this association appeared to be largely driven by the $12 \%$ of cycles from smokers that failed prior to egg retrieval which was more than double the percentage of failures from never (2.0\%) and former smokers (6.3\%). Future observational studies should use the appropriate statistics to account for these effects, particularly when exposures may exert effects early in gestation.

Other than a possible beneficial effect of smoking cessation among male ever-smokers, we did not observe any significant associations when studying male smoking patterns and ART outcomes. Nevertheless, male smoking has been associated with lower semen quality parameters [31] and live birth rates [22] in other epidemiological studies. While our analyses were restricted by power, the fact that former male smokers seemed to more quickly benefit 
from smoking cessation could be explained by the biology of sperm division and differentiation. The mechanism for the observed reduction in cycle failure between clinical pregnancy and live birth due to paternal smoking cessation could be due to lower maternal exposure to second hand smoking and/or a direct paternal exposure [32], although the exact mechanisms remain obscure.

Our study has certain limitations. Due to our design, it may not be possible to extrapolate our results to the general population. However, our cohort of men and women are similar to the population of infertility patients nationwide [33] suggesting that our results might be representative of other couples undergoing ART. While we lacked specific information on the women who declined to participate in the EARTH study, we had a similar smoking prevalence as other ART cohorts in the US [21]. Furthermore, in a previous study on semen quality from MGH, we found that men who refused to participate were comparable to the men who did participate in terms of baseline characteristics [34]. This suggests that the likelihood of selection bias in our study (e.g. smoking men and women being less likely to participate) is low. The women excluded had different baseline characteristics than the included sample; however, our results were similar after performing a sensitivity analysis among all EARTH women and, in fact, some associations gained significance with this increase in power. Another generalizability concern is that in Massachusetts, insurance companies will generally not allow current smokers to have authorization for IVF coverage until cotinine levels are below the non-smoker reference value for 2 months. Therefore self-reported current smokers might not have been smoking for the past two months, which might have attenuated the magnitude of associations towards the null. To address this we assumed a conservative cessation time of at least 2 months for current smokers when modeling time of smoking cessation a continuous exposure. Smoking history was assessed through self-report in our population as opposed to more objective markers like cotinine. While it is possible there was some misclassification of current smokers due to its subjectivity, a person's self-report of smoking is considered reliable [35]. To minimize the possible effect of passive smoking, we adjusted for the partner's smoking history. Although our survival models can account for multiple cycles per women, they are unable to account for the imbalance in number of cycles per couple (e.g. women with more cycles are more likely to fail) [17], yet even when we restricted the analysis to just the first cycle per couple the results were similar. Residual and unmeasured confounding is always possible as this was an observational cohort study; however, we attempted to minimize this by adjusting for number of demographic and lifestyle factors and focusing on a couple analyses. Our main limitation was the small numbers of former and current smokers, reducing our power to detect associations.
Also, the subjects who did smoke reported being mostly "light" or "moderate" smokers in clinical terms. Our study strengths include our prospective design, our detailed assessment of smoking history, and our ability to account for many confounders including the partner's smoking.

\section{Conclusions}

Female smoking intensity was positively associated with the risk of failing in ART cycles between cycle initiation and oocyte retrieval. Among former male smokers, every additional year of smoking cessation was associated with a slightly decreased risk of failing at ART between clinical pregnancy and live birth. Future studies should further investigate the long-term effects of smoking intensity on reproductive potential and take care to use the appropriate analytical approaches to account for failures at early stages of reproduction to reduce the likelihood of survivor bias.

\section{Additional files}

Additional file 1: Table S1. Adjusted mean oocyte yield by smoking patterns among couples with successful egg retrieval. (EARTH Study, $N=225$ couples, 354 ART cycles). (DOCX $17 \mathrm{~kb})$

Additional file 2: Table S2. Adjusted estimated probability of cycles ending in live birth by smoking patterns (EARTH Study, $N=225$ couples, 354 ART cycles). (DOCX $17 \mathrm{~kb}$ )

\begin{abstract}
Abbreviations
95\% Cl: 95\% confidence interval; ART: Assisted reproductive technology; BMI: Body mass index; EARTH: Environment And Reproductive Health study; ET: Embryo transfer; HR: Hazard ratio; ICSI: Intracytoplasmic sperm injection; IVF: In-vitro fertilization; MGH: Massachusetts General Hospital; MIl: Metaphase II oocytes
\end{abstract}

\section{Acknowledgments}

We would like to thank all the patients and personnel involved in the EARTH study from the MGH Fertility Center and in particular thanks to Myra Keller, Ramace Dadd, Patricia Morey, and Tairmee Kangarloo, Harvard T.H. Chan School of Public Health research staff.

\section{Funding}

This work was supported by NIH grants: L50-HD085359 from NICHD, R01ES009718 and P30-ES000002 from NIEHS.

\section{Availability of data and materials}

The datasets generated and/or analyzed during the current study are not publicly available because they contain parts of the study participant's electronical medical records; but are available from the corresponding author on reasonable request.

\section{Authors' contributions}

JCV: first author wrote manuscript and ran statistical analyses. JEC: Help conceived manuscript question and study design, provided manuscript review. PLW: Assisted in the creation of statistical models and manuscript review. JBF: Senior nurse in charge of coordinating participant's enrollment, data entry and manuscript review. TLT: Senior obstetric fertility physician in charge of ART cycles and provided clinical insight and manuscript review. $\mathrm{RH}$ : Conceived initial study question and provided manuscript review. AJG: Senior and corresponding author, provided code and manuscript review as well a principal focus for the analysis. All authors read and approved the final manuscript. 


\section{Authors' information}

For citations and highest academic degree please use: Vanegas JC, MD: Chavarro JE, MD, ScD; Williams PL, PhD; Ford JE, RN, BSN; Toth TL, MD; Hauser R, MD, ScD, MPH; Gaskins AJ, ScD. Affiliations listed on title page.

\section{Competing interests}

The authors declare that they have no competing interests.

\section{Consent for publication}

Not applicable.

\section{Ethics approval and consent to participate}

The study was approved by the Institutional Review Boards of MGH and the Harvard T. H. Chan School of Public Health. All participants provided written informed consent

\section{Author details}

'Department of Nutrition, Harvard T.H. Chan School of Public Health, 665 Huntington Ave., 02115 Boston, MA, USA. ${ }^{2}$ Department of Epidemiology, Harvard T.H. Chan School of Public Health, 665 Huntington Ave., 02115 Boston, MA, USA. ${ }^{3}$ Channing Division of Network Medicine, Department of Medicine, Brigham and Women's Hospital and Harvard Medical School, 181 Longwood Ave., 02115 Boston, MA, USA. ${ }^{4}$ Department of Biostatistics, Harvard School T.H. Chan of Public Health, 665 Huntington Ave., 02115 Boston, MA, USA. ${ }^{5}$ Vincent Obstetrics and Gynecology, Massachusetts General Hospital and Harvard Medical School, 55 Fruit St, 02114 Boston, MA, USA. ${ }^{6}$ Department of Environmental Health, Harvard T.H. Chan School of Public Health, 665 Huntington Ave., 02115 Boston, MA, USA.

\section{Received: 30 November 2016 Accepted: 7 February 2017}

\section{Published online: 20 February 2017}

\section{References}

1. Havelaar AH, Kirk MD, Torgerson PR, Gibb HJ, Hald T, Lake RJ, Praet N, Bellinger DC, de Silva NR, Gargouri N, et al. World Health Organization Global Estimates and Regional Comparisons of the Burden of Foodborne Disease in 2010. PLoS Med. 2015;12:e1001923.

2. World Health Organization (WHO). WHO report on the global tobacco epidemic, 2015: Raising taxes on tobacco. In: Tobacco Free Initiative. Geneva: WHO; 2015.

3. Tong VT, Dietz PM, Morrow B, D'Angelo DV, Farr SL, Rockhill KM, England L. Trends in smoking before, during, and after pregnancy-Pregnancy Risk Assessment Monitoring System, United States, 40 sites, 2000-2010. MMWR Surveill Summ. 2013;62:1-19.

4. Agaku IT, King BA, Husten CG, Bunnell R, Ambrose BK, Hu SS, Holder-Hayes E, Day HR. Tobacco product use among adults-United States, 2012-2013. MMWR Morb Mortal Wkly Rep. 2014;63:542-7.

5. Kaiserman MJ, Rickert WS. Carcinogens in tobacco smoke: benzo[a]pyrene from Canadian cigarettes and cigarette tobacco. Am J Public Health. 1992;82:1023-6.

6. Ding YS, Trommel JS, Yan XJ, Ashley D, Watson CH. Determination of 14 polycyclic aromatic hydrocarbons in mainstream smoke from domestic cigarettes. Environ Sci Technol. 2005;39:471-8.

7. Augood C, Duckitt K, Templeton AA. Smoking and female infertility: a systematic review and meta-analysis. Hum Reprod. 1998;13:1532-9.

8. Jeng HA, Chen YL, Kantaria KN. Association of cigarette smoking with reproductive hormone levels and semen quality in healthy adult men in Taiwan. J Environ Sci Health A Tox Hazard Subst Environ Eng. 2014:49:262-8.

9. Waylen AL, Metwally M, Jones GL, Wilkinson AJ, Ledger WL. Effects of cigarette smoking upon clinical outcomes of assisted reproduction: a metaanalysis. Hum Reprod Update. 2009;15:31-44.

10. Winter E, Wang J, Davies MJ, Norman R. Early pregnancy loss following assisted reproductive technology treatment. Hum Reprod. 2002;17:3220-3.

11. Penzias AS. Recurrent IVF failure: other factors. Fertil Steril. 2012;97:1033-8.

12. Klonoff-Cohen $H$, Natarajan $L$, Marrs $R$, Yee B. Effects of female and male smoking on success rates of IVF and gamete intra-Fallopian transfer. Hum Reprod. 2001:16:1382-90.

13. Cinar O, Dilbaz S, Terzioglu F, Karahalil B, Yucel C, Turk R, Taskin L, Kose SK. Does cigarette smoking really have detrimental effects on outcomes of IVF? Eur J Obstet Gynecol Reprod Biol. 2014;174:106-10.
14. Hauser R, Meeker JD, Duty S, Silva MJ, Calafat AM. Altered semen quality in relation to urinary concentrations of phthalate monoester and oxidative metabolites. Epidemiology. 2006;17:682-91.

15. Pinsky PF, Zhu CS, Kramer BS. Lung cancer risk by years since quitting in 30 + pack year smokers. J Med Screen. 2015;22:151-7.

16. Mok-Lin E, Ehrlich S, Williams PL, Petrozza J, Wright DL, Calafat AM, Ye X, Hauser R. Urinary bisphenol A concentrations and ovarian response among women undergoing IVF. Int J Androl. 2010;33:385-93.

17. Maity A, Williams PL, Ryan L, Missmer SA, Coull BA, Hauser R. Analysis of in vitro fertilization data with multiple outcomes using discrete time-to-event analysis. Stat Med. 2014;33:1738-49.

18. Missmer SA, Pearson KR, Ryan LM, Meeker JD, Cramer DW, Hauser R. Analysis of multiple-cycle data from couples undergoing in vitro fertilization: methodologic issues and statistical approaches. Epidemiology. 2011;22:497-504.

19. Leffondre K, Abrahamowicz M, Siemiatycki J, Rachet B. Modeling smoking history: a comparison of different approaches. Am J Epidemiol. 2002;156:813-23.

20. Neal MS, Hughes EG, Holloway AC, Foster WG. Sidestream smoking is equally as damaging as mainstream smoking on IVF outcomes. Hum Reprod. 2005;20:2531-5.

21. Wright KP, Trimarchi JR, Allsworth J, Keefe D. The effect of female tobacco smoking on IVF outcomes. Hum Reprod. 2006;21:2930-4.

22. Fuentes A, Munoz A, Barnhart K, Arguello B, Diaz M, Pommer R. Recent cigarette smoking and assisted reproductive technologies outcome. Fertil Steril. 2010:93:89-95.

23. Mai Z, Lei M, Yu B, Du H, Liu J. The effects of cigarette smoke extract on ovulation, oocyte morphology and ovarian gene expression in mice. PLoS One. 2014;9:e95945

24. Sadeu JC, Foster WG. Cigarette smoke condensate exposure delays follicular development and function in a stage-dependent manner. Fertil Steril. 2011;95:2410-7.

25. Bolumar F, Olsen J, Boldsen J. Smoking reduces fecundity: a European multicenter study on infertility and subfecundity. The European Study Group on Infertility and Subfecundity. Am J Epidemiol. 1996;143:578-87.

26. Van Voorhis BJ, Syrop CH, Hammitt DG, Dunn MS, Snyder GD. Effects of smoking on ovulation induction for assisted reproductive techniques. Fertil Steril. 1992;58:981-5

27. Freour T, Masson D, Dessolle L, Allaoua D, Dejoie T, Mirallie $S$, Jean M, Barriere $P$. Ovarian reserve and in vitro fertilization cycles outcome according to women smoking status and stimulation regimen. Arch Gynecol Obstet. 2012;285:1177-82.

28. Weigert M, Hofstetter G, Kaipl D, Gottlich H, Krischker U, Bichler K, Poehl M, Feichtinger $W$. The effect of smoking on oocyte quality and hormonal parameters of patients undergoing in vitro fertilization-embryo transfer. J Assist Reprod Genet. 1999;16:287-93.

29. Buckley JP, Keil AP, McGrath LJ, Edwards JK. Evolving methods for inference in the presence of healthy worker survivor bias. Epidemiology. 2015;26:204-12.

30. Hyland A, Piazza KM, Hovey KM, Ockene JK, Andrews CA, Rivard C, Wactawski-Wende J. Associations of lifetime active and passive smoking with spontaneous abortion, stillbirth and tubal ectopic pregnancy: a crosssectional analysis of historical data from the Women's Health Initiative. Tob Control. 2015:24:328-35.

31. Caserta D, Bordi G, Di Segni N, D’Ambrosio A, Mallozzi M, Moscarini M. The influence of cigarette smoking on a population of infertile men and women. Arch Gynecol Obstet. 2013;287:813-8.

32. Venners SA, Wang X, Chen C, Wang L, Chen D, Guang W, Huang A, Ryan L, O'Connor J, Lasley B, et al. Paternal smoking and pregnancy loss: a prospective study using a biomarker of pregnancy. Am J Epidemiol. 2004;159:993-1001.

33. Centers for Disease Control and Prevention (CDC), American Society for Reproductive Medicine (ASRM), Society for Assisted Reproductive Technology (SART). Assisted Reproductive Technology National Summary Report. Atlanta: US Dept of Health and Human Services; 2013.

34. Hauser R, Godfrey-Bailey L, Chen Z. Does the potential for selection bias in semen quality studies depend on study design? Experience from a study conducted within an infertility clinic. Hum Reprod. 2005;20:2579-83.

35. Soulakova JN, Hartman AM, Liu B, Willis GB, Augustine S. Reliability of adult self-reported smoking history: data from the tobacco use supplement to the current population survey 2002-2003 cohort. Nicotine Tob Res. 2012;14:952-60 